HETEROCYCLES, Vol. 79, 2009, pp. 1 - 2. @ The Japan Institute of Heterocyclic Chemistry DOI: $10.3987 / C O M-08-S(D)$ Preface-1

\title{
Preface
}

\section{A Life Dedicated to Chemistry in Nature}

"Our father: a fisherman, scientist, mentor and adventurer, died March 5, 2008..." (An initial sentence of a letter from John's daughters: Kathryn Daly and Shannon Daly Ostrander, to friends of their father). "John W. Daly is a chemist, pharmacologist, and chemical ecologist" (C\&EN/June 26, 2006). Chemistry and biology have been "two separate cultures", yet chemists have increasingly been pursuing biological problems. We can see this excellent evidence in Dr. John W. Daly's distinguished scientific achievements.

In 1959, John and I started our research days as lab-mates at the National Institute of Arthritis and Metabolic Disease (NIH) led by Dr. Bernhard Witkop, Laboratory of Chemistry, Bldg 4, Rm 624. Since then, we have been friends and scientific colleagues over the next three decades. We have visited each other many times for various international projects including one for the Japan Society for the Promotion of Science.

Our mentor Dr. Witkop introduced John to study toxins and requested him to go to Columbia to research frog toxins. John also developed experimental skills in pharmacology while working under the late Nobel laureate, Julius Axelrod. Thus for more than 40 years, he has contributed to a large collection of scientifically interesting new bioactive compounds in nature, and characterized them as both chemical and pharmacological. Remarkably, some of them became extremely important molecular research tools in a wide range of biological fields.

"A Life Dedicated to Chemistry in Nature" was the title of the memorial tribute held in June 12, 2008 at the NIH to honor John's scientific and personal legacy. His world of science was exceedingly diverse and deep. In the following Preface, Dr. Takashi Tokuyama introduces some organic chemical aspects. Further, Dr. Kenneth A. Jacobson et al. contribute a comprehensive review of John's scientific history. ${ }^{1}$ It has been my great pleasure, honor and fortune to share a laboratory with John, and to 
have been associated with him since these early years. His distinguished research activities produced a long list of awards and honors. ${ }^{1}$

This special issue was initially planned to honor him on his $75^{\text {th }}$ birthday. Therefore, we are deeply sad that he is no longer with us. Finally, I am grateful to Mr. Koichi Kametani, President of the Institute, and Professor Keiichiro Fukumoto, Editor, of this memorial publication for him. I would like to thank Dr. Naoki Toyooka for his editing help.

October, 2008

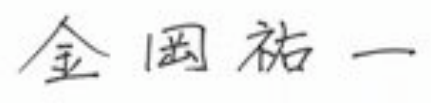

Yuichi Kanaoka

\section{REFERENCES}

1. K. A. Jacobson and K. L. Kirk, “J. W. Daly - An Appreciation”, this volume, pp. 9.

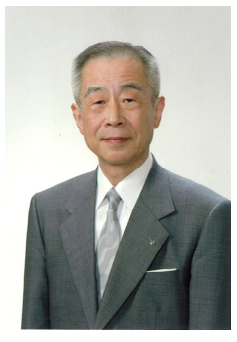

Yuichi Kanaoka, born in 1928 and obtained a B.Ph. in 1950 and a Ph.D. in 1959 in the field of heterocyclic chemistry from University of Tokyo. He joined Hokkaido University in 1956, promoted to full professor in 1966. In 1959-61 he was a research associate at the NIH. He was Dean of Pharmaceutical Faculty and Director of Catalysis Research Center. In 1991 he retired from Hokkaido University, became an Emeritus Professor and moved to Toyama International Institution, where he became Chairman and also President of Toyama College (1993-present) and President of Toyama University of International Studies (2001-07). He was the President of the Pharmaceutical Society of Japan (1994-96) and Member (1994-2003)/ Chairman (2000-03) of the Science Council of Japan - Medical, Dental, and Pharmaceutical Division. His research interests include heterocyclic, synthetic and bioorganic chemistry, and chemical biology. He received the Pharmaceutical Society of Japan Award in 1980, and Millennial Pharmaceutical Scientist Award from the Millennial World Congress of Pharmaceutical Sciences in 2000. 